\title{
Evolution of myeloid leukemia in children with Down syndrome
}

\author{
Satoshi Saida ${ }^{1}$
}

Received: 13 January 2016 / Revised: 9 February 2016 / Accepted: 9 February 2016 / Published online: 24 February 2016

(C) The Japanese Society of Hematology 2016

\begin{abstract}
Children with Down syndrome (DS) have a markedly increased risk of leukemia. They are at particular risk of acute megakaryoblastic leukemia, known as myeloid leukemia associated with DS (ML-DS), the development of which is closely linked to a preceding temporary form of neonatal leukemia called transient abnormal myelopoiesis (TAM). Findings from recent clinical and laboratory studies suggest that constitutional trisomy 21 and GATA1 mutation(s) cause TAM, and that additional genetic alteration(s) including those in epigenetic regulators and signaling molecules are involved in the progression from TAM to ML-DS. Thus, this disease progression represents an important model of multi-step leukemogenesis. The present review focuses on the evolutionary process of TAM to ML-DS, and advances in the understanding of perturbed hematopoiesis in DS with respect to GATA1 mutation and recent findings, including cooperating genetic events, are discussed.
\end{abstract}

Keywords Myeloid leukemia - Down syndrome · Transient leukemia

\section{Introduction}

Down syndrome (DS) results from trisomy 21 and occurs in 1 in 700-1,000 births [1,2]. Although the incidence of neoplasms in DS does not differ significantly from that in

Satoshi Saida

satoshi@kuhp.kyoto-u.ac.jp

1 Department of Pediatrics, Graduate School of Medicine, Kyoto University, 54 Kawahara-cho, Shogoin, Sakyo-ku, Kyoto 606-8507, Japan the general population, the distribution of malignancies is different [3]. Patients with DS show a unique spectrum of malignancies, with a 10- to 20-fold higher risk of acute leukemia and a lower incidence of solid tumors [3, 4]. Death from malignancies other than leukemia is strikingly less common in patients of all ages in DS, and death from leukemia is more likely in children younger than 10 years of age with DS than in those without [5]. The most frequent form of leukemia during childhood, both with and without DS, is acute lymphoblastic leukemia (ALL), and the incidence of ALL in children with DS is approximately 20 -fold higher than in non-DS children [3]. However, the most marked increase in incidence in DS infants is with acute megakaryoblastic leukemia (AMKL), a myeloid malignancy of platelet precursors. The relative risk of developing AMKL is estimated to be 500 times higher in children with DS than in the general population [6]. Unlike AMKL in non-DS patients, most AMKL associated with DS (DSAMKL) patients usually respond well to chemotherapy.

In most cases, DS-AMKL is preceded by a temporary form of megakaryoblastic leukemia unique to newborns with DS and known as transient abnormal myelopoiesis (TAM) or transient leukemia. TAM, a clonal myeloproliferative syndrome, presents in the fetus or a few days after birth and in most cases resolves spontaneously within 3 months [7, 8]. Typically, TAM is characterized by the presence of high numbers of circulating blast cells expressing CD33, CD38, CD117, CD34, CD7, CD56, CD36, $\mathrm{CD} 71$, and $\mathrm{CD} 42 \mathrm{~b}$ [9], which are indistinguishable from blasts observed in DS-AMKL. More often, the clinical presentation of TAM varies from asymptomatic alterations in the blood count to disseminated leukemic infiltration. Clinically severe TAM (liver failure, pleural/pericardial effusion, ascites, renal failure, and coagulopathy) affects $10-30 \%$ of patients with clinically diagnosed TAM, and 
treatment could be required for the patient with these lifethreatening symptoms. A small proportion of these TAM patients will die from their disease, usually due to liver failure/fibrosis $[10,11]$.

After spontaneous remission, $20 \%$ of TAM patients develop myelodysplastic syndrome (MDS) and DS-AMKL within 4 years $[6,12]$. These clinical findings have led many physicians to consider TAM as pre-leukemic and the progression of TAM to DS-AMKL as an attractive model to investigate multi-step leukemogenesis. MDS and DSAMKL were given a special World Health Organization (WHO) sub-classification of "Myeloid leukemia associated with Down syndrome (ML-DS)" because of their unique clinical and biological features [13, 14]. Accordingly, in this paper, the term ML-DS will be used to refer to both entities (DS-related MDS and DS-AMKL).

\section{Abnormal hematopoiesis in DS}

Trisomy 21 can affect hematopoietic cell biology in multiple complex ways [15]. There are $\sim 300$ protein-encoding genes on human chromosome 21 (Hsa21). Some researchers investigated gene expression by human embryonic stem (ES) cells or induced pluripotent stem (iPS) cells derived from primary trisomy 21 samples, showing an intrinsic disturbance of multi-lineage myeloid hematopoiesis at specific stages [16, 17]. Some used a series of transgenic mouse models, including the Tc1 mouse, which contains a copy of most of Hsa21 [18], and other mice containing additional copies of all or part of mouse chromosomal regions corresponding to Hsa21 [19, 20]. These transgenic mouse models provided important insight into the function of a number of genes on chromosome 21 [18-20]. Tc1 mice have macrocytic anemia and increased extramedullary hematopoiesis [18]. Ts65Dn mice, which are trisomic for 104 orthologs of Hsa21 genes, display persistent macrocytosis and develop a myeloproliferative disease characterized by megakaryocyte hyperplasia and dysplasia, and myelofibrosis [19]. Several genes on Hsa21, such as RUNX1, ETS2, $E R G, G A P B A, B A C H 1$, and DYRKIA, encode proteins relating to hematopoiesis and leukemogenesis [9, 21-26]. Further analysis of the models may reveal the effect of chromosome 21 gene dosage on human hematopoiesis and hematological malignancies.

\section{GATA1 mutation induces TAM in DS neonates}

The GATA proteins are a family of transcription factors, three of which are expressed principally in hematopoietic cells (GATA1, GATA2, and GATA3) [27]. GATA1 is encoded on the $\mathrm{X}$ chromosome and is typically present in erythroid, megakaryocytic, mast, and eosinophilic lineage cells [28-31]. GATA2, encoded on chromosome 3, is crucial for the proliferation and survival of early hematopoietic cells, and as a partner of GATA1 is also involved in lineage-specific transcriptional regulation [32]. GATA3, encoded on chromosome 10, plays an essential role in T cell development and immune regulation [33].

GATAl somatic mutations, first identified from a small series of ML-DS patients [34], are present in blast cells both in DS neonates with TAM and children with ML-DS [34-38]. GATA1 mutations disappear when TAM/MLDS enters the remission phase, indicating that these are acquired events [35]. Although it is not yet clear when in fetal development GATA1 mutations arise, the earliest point at which mutations have been identified is 21 weeks of gestation [39].

In a recent UK prospective study, very sensitive nextgeneration sequencing (NGS), which allows the detection of mutant GATAl clones present at very low frequency $(0.3 \%)$, identified GATA1 mutations in $30 \%$ of all neonates with DS, at least half of which were 'silent' with no clinical features of TAM [40]. The reasons for the high frequency of GATA1 mutations in neonates with DS are not known. Of note, none of the 70 neonates without NGS-detected GATA1 mutations in this report developed ML-DS [40]. Accordingly, silent TAM is clinically important because it can subsequently give rise to ML-DS. The study found that $11 \%$ of neonates with a GATA1 mutation (including cases with clinical and silent TAM) went on to develop ML-DS [40]. Although a test for GATAl gene mutation has not yet been standardized in neonates with DS, genetic screening using NGS could be an important tool not only for the diagnosis of TAM, but also for detecting candidates with a potential risk of developing ML-DS.

GATA1 mutations, in the absence of trisomy 21, have not been associated with the development of leukemia. Specific hematopoietic alterations due to GATAl mutation alone include cytopenias [34], Diamond-Blackfan anemia [41], and, in the case of germline GATAl mutations, tri-lineage bone marrow dysplasia [42]. Given that GATAl mutations are not leukemogenic in the absence of constitutional trisomy 21 (cT21), cT21 and mutated GATA1 are thought to be both necessary and sufficient for the generation of TAM [42]. However, clinical findings such as spontaneous remission in TAM suggest that mutation in GATAl is necessary, but insufficient for the development of ML-DS.

Originally, it was believed that the loss of the N-terminal transactivation domain (exon 1) reduces the activity of GATA1 in regulating the terminal differentiation of megakaryocytes, thereby leading to accumulation of poorly differentiated megakaryocytic progenitors [34]. However, most of the mutations are found in exon 2 of the GATAl gene, with a minority in exon 3 or at the intronic boundary 
of exon 1 and 2, and lead to expression of N-terminally truncated GATA1 (GATA1s) protein [34, 43]. GATA1s lacks the N-terminal transactivation domain, but retains both DNA-binding zinc fingers [34]. The presence of GATA1s is thought to impair GATA1-mediated regulation of other transcription factors, including GATA2, MYB, MYC, and IKAROS family zinc finger 1 (IKZF1), in fetal megakaryocytes [44]. GATA1 knockdown mice accumulate immature erythroid progenitors [45], and when transgenically 'rescued' with GATA1s these mice have an abnormal accumulation of CD41+ megakaryocytic progenitors [46]. Furthermore, forced expression of GATA1s in fetal hematopoietic progenitors from mice bearing wild-type GATA1 causes marked expansion of megakaryoblastic progenitors [25]. Toki et al. [47] also revealed that GATA1s promotes megakaryocyte proliferation more profoundly than that induced by GATA1 deficiency, supporting a gain-offunction mechanism for GATA1s. Collectively, although both GATA1 dysfunction and GATA1s expression are important in the development of TAM, GATA1 dysfunction alone does not appear to be sufficient, and it may be overexpression of GATA1s that contributes to the development of DS-related myeloid disorders. Interestingly, although how GATA1s contributes to the TAM phenotype remains unclear, GATA1 mutation type and GATA1s expression levels are significantly associated with a risk of progression to ML-DS [45, 48].

\section{Genetic heterogeneity in TAM}

Cancer arises through an evolutionary process of somatic mutation and selection [49]. This evolutionary process, clonal evolution, is associated with significant intratumoral heterogeneity [50]. Recently, new genomic technologies have led to a better understanding of the complex clonal architecture of leukemia [51-54].

\section{TAM xenograft model}

Mouse models in which primary human leukemic cells were transplanted into immunodeficient hosts provide significant clues to advance our understanding of the pathogenesis of human leukemia [51-53, 55]. However, xenograft models using primary patient samples from the pre-leukemic phase have been rarely reported. We hypothesize that TAM xenograft models would be an attractive method for investigating the evolutionary process of leukemia. In contrast to a previous study in which TAM cells showed a limited ability to expand in immunodeficient mice [56], we established a xenograft model of TAM using NOD/Shi-scid, IL-2R $\gamma$ null (NOG) mice, which mimicked the progression of TAM to DS-AMKL [57]. The presence of the same GATAl mutation was confirmed in the primary TAM cells and the engrafted cells in the NOG mice. These TAM-derived cells were morphologically similar to the primary TAM cells obtained from the patients. Flow cytometric analysis of surface antigens detected an expression pattern consistent with that observed in primary cells of TAM patients. Interestingly, in this model, the engrafted cells predominantly comprised a single genetically defined subclone of the TAM patient cells (Fig. 1a).

\section{Genetically heterogeneous subclones with varying repopulating capacity expanded in the TAM xenograft model}

Of engrafted TAM cells derived from three patients (\#1, \#2, and \#9), only cells from patient \#1 successfully engrafted into secondary as well as tertiary recipients, indicating that some TAM clones have long-term self-renewal capacity, a characteristic of leukemia, at least in xenografts. Interestingly, TAM patient \#1 went on to develop ML-DS at the age of 1 year, 10 months after the TAM cell sample was taken, whereas the other patients did not. Compared with TAM, additional chromosomal alterations and copy number alterations (CNAs) are frequently observed in ML-DS $[8,58-60]$, and primary samples from TAM patient \#1 had no CNAs other than the gain of chromosome 21 (Fig. 1b). However, serial transplantations demonstrated that TAMderived cells from patient \#1 acquired various CNAs, a hallmark of ML-DS, and showed diverse repopulating capacity in xenografts (Fig. 1b). Breakpoint-specific PCR analysis demonstrated that TAM cells with these CNAs were already present as a minor population in the original sample from patient \#1. In addition, engrafted cells derived from this primary patient sample revealed the presence of another minor GATAl mutation that was not detected by conventional sequencing in the original cell sample (Fig. 1b). These findings suggest that pre-leukemic TAM is far more genetically heterogeneous than first suspected.

\section{Clinical findings}

Ahmed et al. [35] reported that multiple separate GATAl mutant clones could occur in an individual TAM patient. In addition, $\mathrm{Xu}$ et al. [61] reported that ML-DS can arise from a minor TAM clone with a GATA1 mutation that is distinct from that of the patient's major TAM clone. Recently, whole exome/genome sequencing of paired TAM and ML-DS samples also indicated that ML-DS may develop not only from a major GATAl mutant subclone present in the TAM phase, but also from a minor mutant GATAl clone [62]. Furthermore, whole exome sequencing results 
A

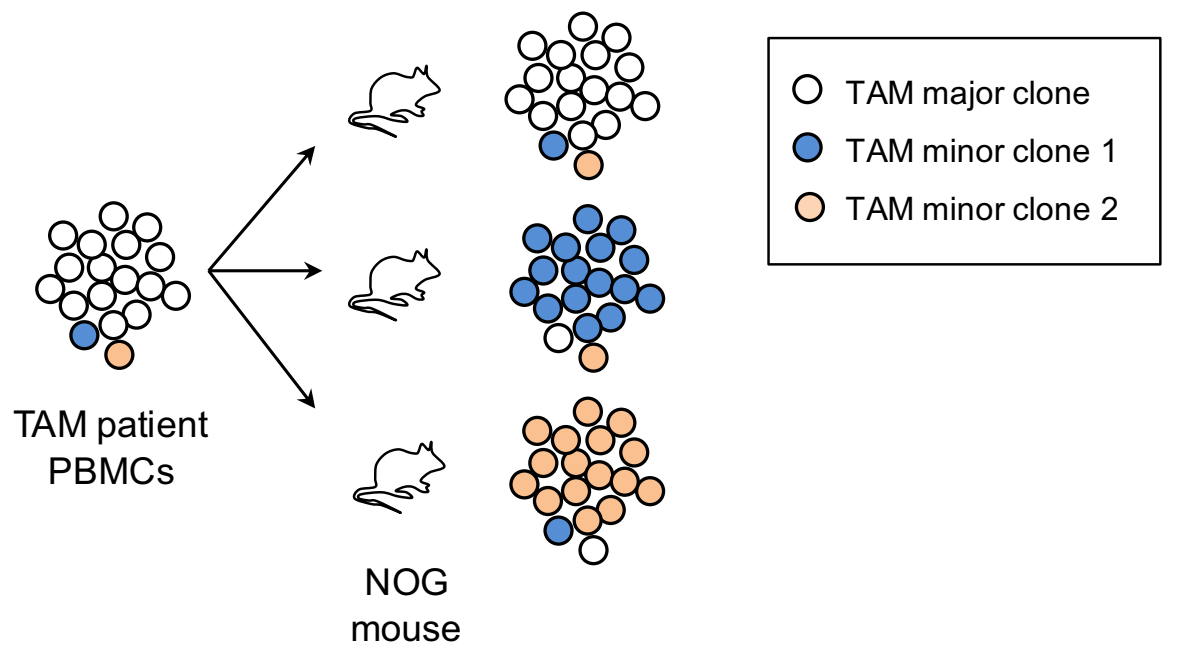

B

Patient \#1

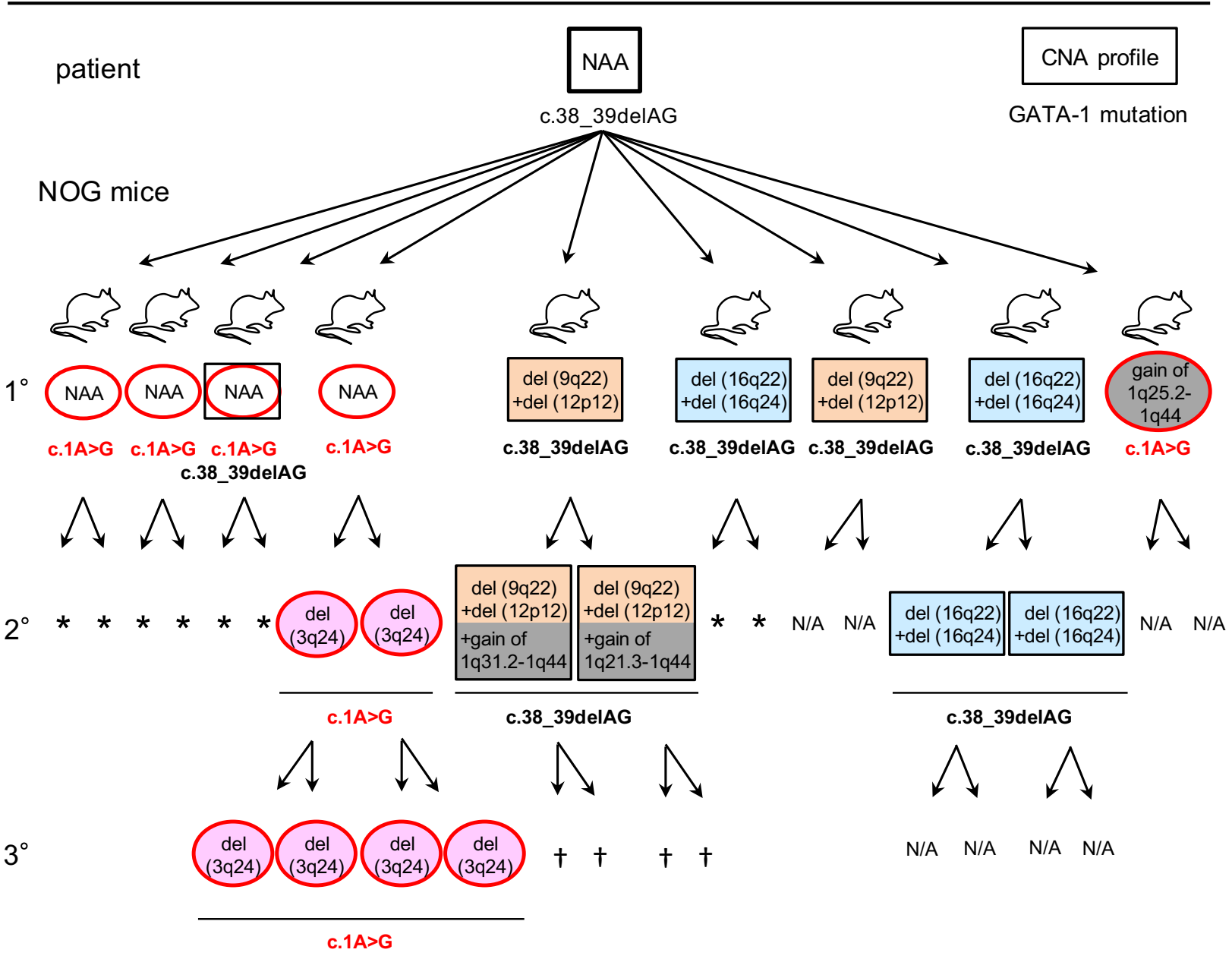

also suggest the presence of intratumoral heterogeneity in the majority of DS-AMKL cases [62]. Thus, recent new genomic and NGS technologies are together enabling the genetic heterogeneity of TAM and ML-DS to be unraveled.

\section{Additional genomic events induce ML-DS}

Estimates of the risk of ML-DS following TAM vary from $\sim 5 \%$ in the recent prospective study to $30 \%$ in 
4 Fig. 1 a Schema of experimental design for tracking primary TAM clones in xenografts. Peripheral blood mononuclear cells (PBMCs) were obtained from TAM patients and transplanted into irradiated NOG mice via the tail vein. TAM major clone white circle; TAM minor clone 1 blue circle; TAM minor clone 2 orange circle. b Summary of the serial transplantation of TAM cells of patient \#1 who developed ML-DS at 1 year of age, and the results of CNA profiling and GATA1 mutation analysis. The original patient sample had a single GATA1 mutation, c.38G_39delAG, and no additional CNAs. Diverse subpopulations with or without additional CNAs expanded in each recipient. GATAl mutation analysis showed two distinct mutations in recipients, one identical to that of the original patient (c.38_39delAG) and a different mutation $(\mathrm{c} .1 \mathrm{~A}>\mathrm{G})$. The mice harboring cells with the original mutation (c.38_39delAG) are depicted as black rectangles, and the mice with cells harboring the other mutation (c. $1 \mathrm{~A}>\mathrm{G}$ ) are depicted as red ovals; the CNA profile is given within the graphic. The GATA1 mutation is indicated below the symbol. NAA no additional alteration, N/A not assessed due to low blast cell count, ${ }^{\dagger}$ death of recipient before analysis. Asterisk represents no engraftment. This research was originally published in Ref. [57]

retrospective studies of clinically diagnosed TAM $[8,11$, 40], and analysis of paired samples from the same patient found the identical GATAl mutation in both pre-leukemic (TAM) and leukemic (ML-DS) stages [11, 37, 43].
Because only 5-30 \% of TAM cases progress to ML-DS, additional genetic events besides GATAl mutation are suspected to be involved in the progression [63]. For example, recurrent additional cytogenetic abnormalities are commonly observed during disease progression $[8,43,58,59]$. Classically reported, the development of ML-DS is significantly correlated with karyotypic abnormalities such as $\operatorname{dup}(1 \mathrm{q}), \operatorname{del}(6 q), \operatorname{del}(7 \mathrm{p}), \operatorname{dup}(7 q),+8,+11$, and $\operatorname{del}(16 q)$, which are rarely observed in the TAM phase $[8,58,59]$. However, evidence for a role of chromosomal instability in DS malignancies is conflicting, and whether these chromosomal abnormalities cause the leukemic phenotype is unclear [64, 65].

On the other hand, many studies have elucidated the details of genomic changes during ML-DS progression. Initially, a few candidate tumor-associated genes were identified in the progression to ML-DS [66-68]. NGS studies of TAM/ML-DS now reveal a high frequency of mutations in cohesin, CCCTC-binding factor (CTCF), and other epigenetic regulators $[62,69]$. While the normal function of these proteins is incompletely understood, roles for cohesin and CTCF are emerging as coordinators of long-range

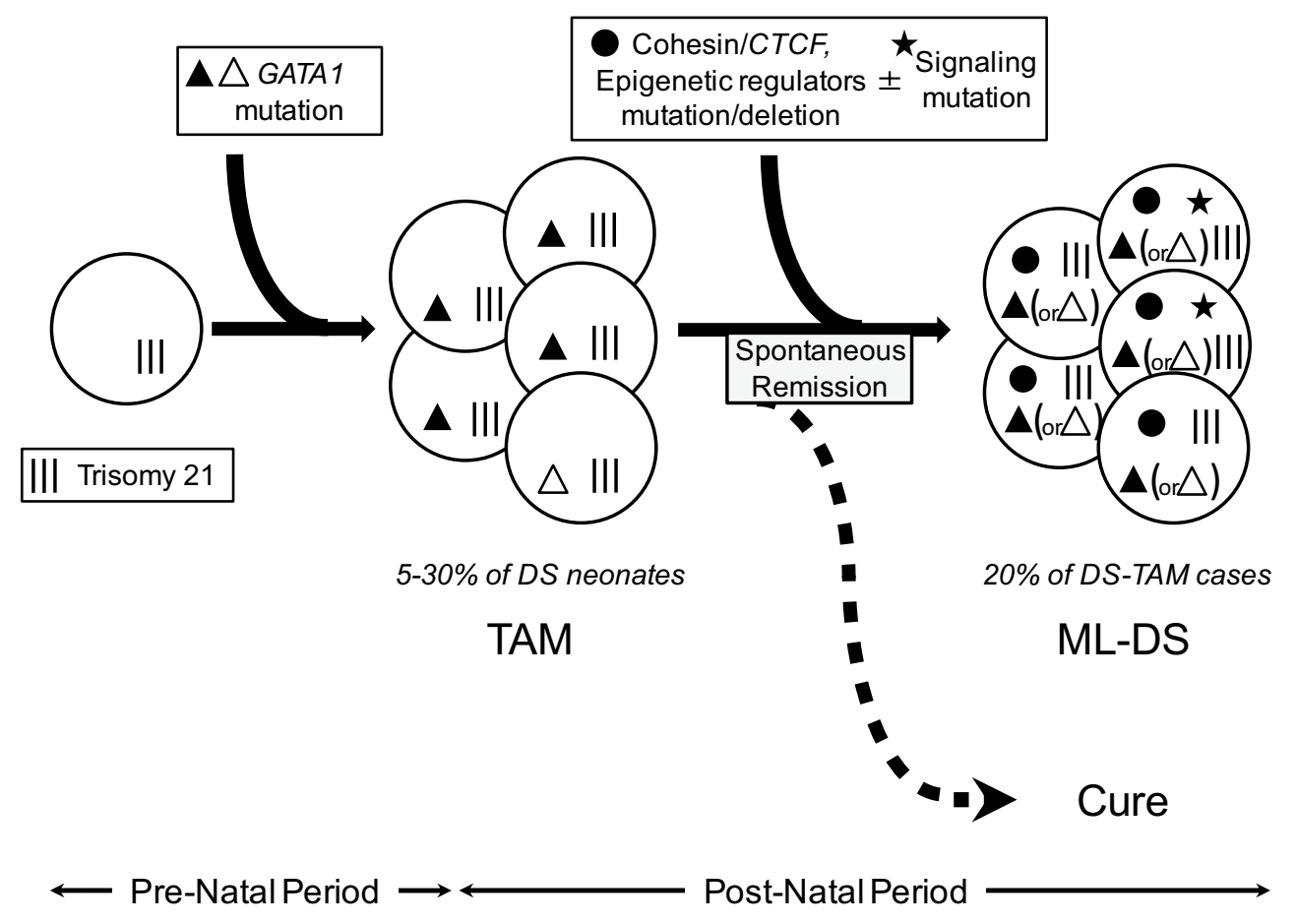

Fig. 2 The proposed model of ML-DS pathogenesis. In the setting of trisomy 21, an in utero truncating mutation in GATA1 induces TAM in the neonatal period. At birth, multiple clones with different GATA1 mutations (indicated by filled triangles and open triangles) might be present, whereas one GATA1 mutant clone is predominant in most cases of overt TAM. Although TAM resolves spontaneously in most cases, residual cells acquire additional cooperating/driver mutations (cohesin, CTCF, other epigenetic regulators, and kinase-signal- ing molecules) leading to overt ML-DS within 4 years. ML-DS originates from one of the multiple GATA1 mutant clones present in the TAM phase, usually representing the progeny of the largest (sometimes from the minor) subpopulation. Trisomy 21 bars; GATA1 mutation filled triangles and open triangles; cohesin, CTCF, and other epigenetic regulator mutation filled circles; kinase-signaling molecule mutation filled stars. Figure adapted from Ref. [62] 
interactions between genomic regulatory elements [70], and for epigenetic regulators, such as EZH2, in regulating various epigenetic events [71].

Mutations are also observed in members of signaling pathways, such as the JAK family of kinases, MPL, SH2B3, and multiple RAS pathway genes $[62,69]$. The variant allele frequency (VAF) of mutations in cohesin components, $\mathrm{CTCF}$, and EZH2 implies that their role is in the early stage of ML-DS development [62]. By contrast, the RAS pathway and other signaling mutations were more likely to represent subclonal mutations, which were typically preceded by mutations in cohesin components, CTCF, and EZH2 and were involved in the evolution of multiple DS-AMKL subclones [62]. Importantly, these genomic analyses of ML-DS confirm that it evolves from the cells responsible for TAM.

\section{Conclusions and future directions}

NGS-based studies unraveled the genomic landscape of DSrelated myeloid disorders [72, 73]. ML-DS is shaped by multiple rounds of acquisition of new mutations and clonal selection (Fig. 2). In the setting of trisomy 21, GATA1 mutation(s) causes TAM. Although most TAM resolves spontaneously, further mutation in cohesin, CTCF, EZH2, or other epigenetic regulators in residual TAM cells occurs with or without mutation of signal-transducing molecules, leading to ML-DS (Fig. 2). Recent studies reveal that genetic intratumoral heterogeneity was evident not only in the ML-DS, but also in the TAM phase [57, 62], and that ML-DS originated from one of the multiple subclones present in the TAM phase of the disease. NGS methodology also showed the precise frequency of GATAl mutation in DS [40] and may be used in the future to make diagnostic and treatment decisions in DS-related myeloid disorders.

The recent findings of mutations in epigenetic regulators in ML-DS suggest that epigenetics also play a role in the development of ML-DS. Indeed, Malinge et al. [74] showed that TAM and ML-DS are associated with sequential epigenetic changes. They showed that trisomy 21 led to global hypomethylation and that TAM samples featured new, focal gains of DNA methylation. However, the transcriptome and epigenome of TAM samples compared with ML-DS in their report were very similar [74]. A crucial role for mutations in epigenetic regulators in the evolutionary process of TAM to ML-DS remains unclear.

Furthermore, a number of key questions remain unanswered. Why are mutations in cohesin and CTCF especially common in ML-DS? How do these mutations contribute to the etiology of ML-DS and the relationship with GATA1 mutation? Why does TAM resolve spontaneously? How do residual TAM cells survive? How can we predict and prevent the incidence of ML-DS in TAM patients? These questions will continue to drive investigations into this curious disorder.

Acknowledgments This work was supported by the Japan Society for the Promotion of Science (JSPS) KAKENHI Grant-in-Aid for Young Scientists (B) Research Project Number 26860795.

\section{Compliance with ethical standards}

Conflict of interest The author has no conflict of interest to declare.

\section{References}

1. Parker SE, Mai CT, Canfield MA, Rickard R, Wang Y, Meyer $\mathrm{RE}$, et al. Updated National Birth Prevalence estimates for selected birth defects in the United States, 2004-2006. Birth Defects Res A Clin Mol Teratol. 2010;88(12):1008-16.

2. Roizen NJ, Patterson D. Down's syndrome. Lancet. 2003;361(9365):1281-9.

3. Hasle H, Clemmensen IH, Mikkelsen M. Risks of leukaemia and solid tumours in individuals with Down's syndrome. Lancet. 2000;355(9199):165-9.

4. Fong CT, Brodeur GM. Down's syndrome and leukemia: epidemiology, genetics, cytogenetics and mechanisms of leukemogenesis. Cancer Genet Cytogenet. 1987;28(1):55-76.

5. Yang Q, Rasmussen SA, Friedman JM. Mortality associated with Down's syndrome in the USA from 1983 to 1997: a populationbased study. Lancet. 2002;359(9311):1019-25.

6. Zipursky A, Poon A, Doyle J. Leukemia in Down syndrome: a review. Pediatr Hematol Oncol. 1992;9(2):139-49.

7. Pine SR, Guo Q, Yin C, Jayabose S, Druschel CM, Sandoval C. Incidence and clinical implications of GATA1 mutations in newborns with Down syndrome. Blood. 2007;110(6):2128-31.

8. Massey GV, Zipursky A, Chang MN, Doyle JJ, Nasim S, Taub $\mathrm{JW}$, et al. A prospective study of the natural history of transient leukemia (TL) in neonates with Down syndrome (DS): Children's Oncology Group (COG) study POG-9481. Blood. 2006;107(12):4606-13.

9. Roy A, Roberts I, Norton A, Vyas P. Acute megakaryoblastic leukaemia (AMKL) and transient myeloproliferative disorder (TMD) in Down syndrome: a multi-step model of myeloid leukaemogenesis. Br J Haematol. 2009;147(1):3-12.

10. Klusmann JH, Creutzig U, Zimmermann M, Dworzak M, Jorch $\mathrm{N}$, Langebrake $\mathrm{C}$, et al. Treatment and prognostic impact of transient leukemia in neonates with Down syndrome. Blood. 2008;111(6):2991-8.

11. Gamis AS, Smith FO. Transient myeloproliferative disorder in children with Down syndrome: clarity to this enigmatic disorder. Br J Haematol. 2012;159(3):277-87.

12. Hitzler JK. Acute megakaryoblastic leukemia in Down syndrome. Pediatr Blood Cancer. 2007;49(7 Suppl):1066-9.

13. Hasle H, Niemeyer CM, Chessells JM, Baumann I, Bennett JM, Kerndrup G, et al. A pediatric approach to the WHO classification of myelodysplastic and myeloproliferative diseases. Leukemia. 2003;17(2):277-82.

14. Vardiman JW, Thiele J, Arber DA, Brunning RD, Borowitz MJ, Porwit A, et al. The 2008 revision of the World Health Organization (WHO) classification of myeloid neoplasms and acute leukemia: rationale and important changes. Blood. 2009;114(5):937-51.

15. Roberts I, O'Connor D, Roy A, Cowan G, Vyas P. The impact of trisomy 21 on foetal haematopoiesis. Blood Cells Mol Dis. 2013;51(4):277-81. 
16. Maclean GA, Menne TF, Guo G, Sanchez DJ, Park IH, Daley $\mathrm{GQ}$, et al. Altered hematopoiesis in trisomy 21 as revealed through in vitro differentiation of isogenic human pluripotent cells. Proc Natl Acad Sci USA. 2012;109(43):17567-72.

17. Chou ST, Byrska-Bishop M, Tober JM, Yao Y, Vandorn D, Opalinska JB, et al. Trisomy 21-associated defects in human primitive hematopoiesis revealed through induced pluripotent stem cells. Proc Natl Acad Sci USA. 2012;109(43):17573-8.

18. Alford KA, Slender A, Vanes L, Li Z, Fisher EM, Nizetic D, et al. Perturbed hematopoiesis in the Tc1 mouse model of Down syndrome. Blood. 2010;115(14):2928-37.

19. Kirsammer G, Jilani S, Liu H, Davis E, Gurbuxani S, Le Beau MM, et al. Highly penetrant myeloproliferative disease in the Ts65Dn mouse model of Down syndrome. Blood. 2008;111(2):767-75.

20. Carmichael CL, Majewski IJ, Alexander WS, Metcalf D, Hilton DJ, Hewitt CA, et al. Hematopoietic defects in the Ts1Cje mouse model of Down syndrome. Blood. 2009;113(9):1929-37.

21. Ichikawa M, Yoshimi A, Nakagawa M, Nishimoto N, WatanabeOkochi N, Kurokawa M. A role for RUNX1 in hematopoiesis and myeloid leukemia. Int J Hematol. 2013;97(6):726-34.

22. Papas TS, Watson DK, Sacchi N, Fujiwara S, Seth AK, Fisher RJ, et al. ETS family of genes in leukemia and Down syndrome. Am J Med Genet Suppl. 1990;7:251-61.

23. Malinge S, Bliss-Moreau M, Kirsammer G, Diebold L, Chlon $\mathrm{T}$, Gurbuxani S, et al. Increased dosage of the chromosome 21 ortholog Dyrk1a promotes megakaryoblastic leukemia in a murine model of Down syndrome. J Clin Invest. 2012;122(3):948-62.

24. Gardiner K. Transcriptional dysregulation in Down syndrome: predictions for altered protein complex stoichiometries and post-translational modifications, and consequences for learning/ behavior genes ELK, CREB, and the estrogen and glucocorticoid receptors. Behav Genet. 2006;36(3):439-53.

25. Salek-Ardakani S, Smooha G, de Boer J, Sebire NJ, Morrow M, Rainis L, et al. ERG is a megakaryocytic oncogene. Cancer Res. 2009;69(11):4665-73.

26. Malinge $S$, Izraeli $S$, Crispino JD. Insights into the manifestations, outcomes, and mechanisms of leukemogenesis in Down syndrome. Blood. 2009;113(12):2619-28.

27. Gao J, Chen YH, Peterson LC. GATA family transcriptional factors: emerging suspects in hematologic disorders. Exp Hematol Oncol. 2015;4:28.

28. Weiss MJ, Orkin SH. Transcription factor GATA-1 permits survival and maturation of erythroid precursors by preventing apoptosis. Proc Natl Acad Sci USA. 1995;92(21):9623-7.

29. Ferreira R, Ohneda K, Yamamoto M, Philipsen S. GATA1 function, a paradigm for transcription factors in hematopoiesis. Mol Cell Biol. 2005;25(4):1215-27.

30. Zon LI, Yamaguchi Y, Yee K, Albee EA, Kimura A, Bennett JC, et al. Expression of mRNA for the GATA-binding proteins in human eosinophils and basophils: potential role in gene transcription. Blood. 1993;81(12):3234-41.

31. Hirasawa R, Shimizu R, Takahashi S, Osawa M, Takayanagi S, Kato Y, et al. Essential and instructive roles of GATA factors in eosinophil development. J Exp Med. 2002;195(11):1379-86.

32. Vicente C, Conchillo A, Garcia-Sanchez MA, Odero MD. The role of the GATA2 transcription factor in normal and malignant hematopoiesis. Crit Rev Oncol Hematol. 2012;82(1):1-17.

33. Tindemans I, Serafini N, Di Santo JP, Hendriks RW. GATA-3 function in innate and adaptive immunity. Immunity. 2014;41(2):191-206.

34. Wechsler J, Greene M, McDevitt MA, Anastasi J, Karp JE, Le Beau MM, et al. Acquired mutations in GATA1 in the megakaryoblastic leukemia of Down syndrome. Nat Genet. 2002;32(1):148-52.
35. Ahmed M, Sternberg A, Hall G, Thomas A, Smith O, O'Marcaigh A, et al. Natural history of GATA1 mutations in Down syndrome. Blood. 2004;103(7):2480-9.

36. Groet J, McElwaine S, Spinelli M, Rinaldi A, Burtscher I, Mulligan $\mathrm{C}$, et al. Acquired mutations in GATA1 in neonates with Down's syndrome with transient myeloid disorder. Lancet. 2003;361(9369):1617-20.

37. Rainis L, Bercovich D, Strehl S, Teigler-Schlegel A, Stark B, Trka J, et al. Mutations in exon 2 of GATA1 are early events in megakaryocytic malignancies associated with trisomy 21 . Blood. 2003;102(3):981-6.

38. Xu G, Nagano M, Kanezaki R, Toki T, Hayashi Y, Taketani $\mathrm{T}$, et al. Frequent mutations in the GATA-1 gene in the transient myeloproliferative disorder of Down syndrome. Blood. 2003;102(8):2960-8.

39. Taub JW, Mundschau G, Ge Y, Poulik JM, Qureshi F, Jensen T, et al. Prenatal origin of GATA1 mutations may be an initiating step in the development of megakaryocytic leukemia in Down syndrome. Blood. 2004;104(5):1588-9.

40. Roberts I, Alford K, Hall G, Juban G, Richmond H, Norton A, et al. GATA1-mutant clones are frequent and often unsuspected in babies with Down syndrome: identification of a population at risk of leukemia. Blood. 2013;122(24):3908-17.

41. Sankaran VG, Ghazvinian R, Do R, Thiru P, Vergilio JA, Beggs $\mathrm{AH}$, et al. Exome sequencing identifies GATA1 mutations resulting in Diamond-Blackfan anemia. J Clin Invest. 2012;122(7):2439-43.

42. Hollanda LM, Lima CS, Cunha AF, Albuquerque DM, Vassallo $\mathrm{J}$, Ozelo MC, et al. An inherited mutation leading to production of only the short isoform of GATA-1 is associated with impaired erythropoiesis. Nat Genet. 2006;38(7):807-12.

43. Hitzler JK, Cheung J, Li Y, Scherer SW, Zipursky A. GATA1 mutations in transient leukemia and acute megakaryoblastic leukemia of Down syndrome. Blood. 2003;101(11):4301-4.

44. Li Z, Godinho FJ, Klusmann JH, Garriga-Canut M, Yu C, Orkin SH. Developmental stage-selective effect of somatically mutated leukemogenic transcription factor GATA1. Nat Genet. 2005;37(6):613-9.

45. Shimizu R, Kuroha T, Ohneda O, Pan X, Ohneda K, Takahashi $\mathrm{S}$, et al. Leukemogenesis caused by incapacitated GATA-1 function. Mol Cell Biol. 2004;24(24):10814-25.

46. Shimizu R, Kobayashi E, Engel JD, Yamamoto M. Induction of hyperproliferative fetal megakaryopoiesis by an N-terminally truncated GATA1 mutant. Genes Cells. 2009;14(9):1119-31.

47. Toki T, Kanezaki R, Kobayashi E, Kaneko H, Suzuki M, Wang $\mathrm{R}$, et al. Naturally occurring oncogenic GATA1 mutants with internal deletions in transient abnormal myelopoiesis in Down syndrome. Blood. 2013;121(16):3181-4.

48. Kanezaki R, Toki T, Terui K, Xu G, Wang R, Shimada A, et al. Down syndrome and GATA1 mutations in transient abnormal myeloproliferative disorder: mutation classes correlate with progression to myeloid leukemia. Blood. 2010;116(22):4631-8.

49. Nowell PC. The clonal evolution of tumor cell populations. Science. 1976;194(4260):23-8.

50. Shackleton M, Quintana E, Fearon ER, Morrison SJ. Heterogeneity in cancer: cancer stem cells versus clonal evolution. Cell. 2009;138(5):822-9.

51. Notta F, Mullighan CG, Wang JCY, Poeppl A, Doulatov S, Phillips LA, et al. Evolution of human BCR-ABL1 lymphoblastic leukaemia-initiating cells. Nature. 2011;469(7330):362-7.

52. Anderson K, Lutz C, van Delft FW, Bateman CM, Guo Y, Colman SM, et al. Genetic variegation of clonal architecture and propagating cells in leukaemia. Nature. 2011;469(7330):356-61.

53. Clappier E, Gerby B, Sigaux F, Delord M, Touzri F, Hernandez $\mathrm{L}$, et al. Clonal selection in xenografted human $\mathrm{T}$ cell acute 
lymphoblastic leukemia recapitulates gain of malignancy at relapse. J Exp Med. 2011;208(4):653-61.

54. Welch JS, Ley TJ, Link DC, Miller CA, Larson DE, Koboldt DC, et al. The origin and evolution of mutations in acute myeloid leukemia. Cell. 2012;150(2):264-78.

55. Bonnet D, Dick JE. Human acute myeloid leukemia is organized as a hierarchy that originates from a primitive hematopoietic cell. Nat Med. 1997;3(7):730-7.

56. Chen J, Li Y, Doedens M, Wang P, Shago M, Dick J, et al. Functional differences between myeloid leukemia-initiating and transient leukemia cells in Down's syndrome. Leukemia. 2010;24(5):1012-7.

57. Saida S, Watanabe K, Sato-Otsubo A, Terui K, Yoshida K, Okuno $\mathrm{Y}$, et al. Clonal selection in xenografted TAM recapitulates the evolutionary process of myeloid leukemia in Down syndrome. Blood. 2013;121(21):4377-87.

58. Forestier E, Izraeli S, Beverloo B, Haas O, Pession A, Michalova $\mathrm{K}$, et al. Cytogenetic features of acute lymphoblastic and myeloid leukemias in pediatric patients with Down syndrome: an iBFM-SG study. Blood. 2008;111(3):1575-83.

59. Hayashi Y, Eguchi M, Sugita K, Nakazawa S, Sato T, Kojima S, et al. Cytogenetic findings and clinical features in acute leukemia and transient myeloproliferative disorder in Down's syndrome. Blood. 1988;72(1):15-23.

60. Silva ML, do Socorro Pombo-de-Oliveira M, Raimondi SC, Mkrtchyan H, Abdelhay E, de Figueiredo AF, et al. Unbalanced chromosome 1 abnormalities leading to partial trisomy $1 \mathrm{q}$ in four infants with Down syndrome and acute megakaryocytic leukemia. Mol Cytogenet. 2009;2:7.

61. Xu G, Kato K, Toki T, Takahashi Y, Terui K, Ito E. Development of acute megakaryoblastic leukemia from a minor clone in a Down syndrome patient with clinically overt transient myeloproliferative disorder. J Pediatr Hematol Oncol. 2006;28(10):696-8.

62. Yoshida K, Toki T, Okuno Y, Kanezaki R, Shiraishi Y, SatoOtsubo A, et al. The landscape of somatic mutations in Down syndrome-related myeloid disorders. Nat Genet. 2013;45(11):1293-9.
63. Vyas P, Crispino JD. Molecular insights into Down syndromeassociated leukemia. Curr Opin Pediatr. 2007;19(1):9-14.

64. Ganmore I, Smooha G, Izraeli S. Constitutional aneuploidy and cancer predisposition. Hum Mol Genet. 2009;18(R1):R84-93.

65. Nizetic D, Groet J. Tumorigenesis in Down's syndrome: big lessons from a small chromosome. Nat Rev Cancer. 2012;12(10):721-32.

66. Malkin D, Brown EJ, Zipursky A. The role of p53 in megakaryocyte differentiation and the megakaryocytic leukemias of Down syndrome. Cancer Genet Cytogenet. 2000;116(1):1-5.

67. Norton A, Fisher C, Liu H, Wen Q, Mundschau G, Fuster JL, et al. Analysis of JAK3, JAK2, and C-MPL mutations in transient myeloproliferative disorder and myeloid leukemia of Down syndrome blasts in children with Down syndrome. Blood. 2007;110(3):1077-9.

68. Malinge S, Ragu C, Della-Valle V, Pisani D, Constantinescu SN, Perez C, et al. Activating mutations in human acute megakaryoblastic leukemia. Blood. 2008;112(10):4220-6.

69. Nikolaev SI, Santoni F, Vannier A, Falconnet E, Giarin E, Basso $\mathrm{G}$, et al. Exome sequencing identifies putative drivers of progression of transient myeloproliferative disorder to AMKL in infants with Down syndrome. Blood. 2013;122(4):554-61.

70. Merkenschlager M, Odom DT. CTCF and cohesin: linking gene regulatory elements with their targets. Cell. 2013;152(6):1285-97.

71. Vire E, Brenner C, Deplus R, Blanchon L, Fraga M, Didelot C, et al. The Polycomb group protein EZH2 directly controls DNA methylation. Nature. 2006;439(7078):871-4.

72. Gruber TA, Downing JR. The biology of pediatric acute megakaryoblastic leukemia. Blood. 2015;126(8):943-9.

73. Mateos MK, Barbaric D, Byatt S-A, Sutton R, Marshall GM. Down syndrome and leukemia: insights into leukemogenesis and translational targets. Transl Pediatr. 2015;4(2):76-92.

74. Malinge S, Chlon T, Dore LC, Ketterling RP, Tallman MS, Paietta E, et al. Development of acute megakaryoblastic leukemia in Down syndrome is associated with sequential epigenetic changes. Blood. 2013;122(14):e33-43. 